\title{
Reflets
}

Revue d'intervention sociale et communautaire

\section{Les multiples visages du VIH-sida. Représentations, expériences et intervention}

\section{Christine Francoeur}

Volume 17, numéro 2, automne 2011

URI : https://id.erudit.org/iderudit/1012133ar

DOI : https://doi.org/10.7202/1012133ar

Aller au sommaire du numéro

Éditeur(s)

Reflets, Revue d'intervention sociale et communautaire

ISSN

1203-4576 (imprimé)

1712-8498 (numérique)

Découvrir la revue

Citer cet article

Francoeur, C. (2011). Les multiples visages du VIH-sida. Représentations, expériences et intervention. Reflets, 17(2), 150-162.

https://doi.org/10.7202/1012133ar d'utilisation que vous pouvez consulter en ligne.

https://apropos.erudit.org/fr/usagers/politique-dutilisation/ 


\title{
Les multiples visages du VIH-sida. Représentations, expériences et intervention
}

\author{
Christine Francoeur \\ Maîtrise en service social, Université d'Ottawa
}

\section{Introduction $^{1}$}

Trente années se sont écoulées depuis que le VIH/sida a fait ses premières victimes en Amérique du Nord. Au début, personne ne se doutait qu'en trois décennies près de 30 millions d'individus en mourraient. Mais en trente ans, les personnes aux prises avec le VIH/sida ont vécu plusieurs changements. Grâce aux médicaments aujourd'hui disponibles, le VIH/sida est devenu une maladie chronique et les personnes qui en sont atteintes connaissent une qualité de vie qui ne leur était pas accessible auparavant. L'évolution des connaissances médicales permet même à des femmes séropositives d'avoir des enfants.

Le présent article dresse d'abord un bref historique du VIH/ sida et propose un survol des représentations sociales qui s'y sont greffées à la suite des discours scientifiques et médiatiques publiés en début d'épidémie. Puis, afin de mieux comprendre l'enjeu du $\mathrm{VIH} /$ sida, suivra une synthèse de mon mémoire de maitrise en service social, mémoire intitulé Donner la vie : le parcours de mères séropositives - L'expérience de maternité pour des femmes d'origine subsaharienne. Une attention particulière sera enfin portée à mes résultats afin d'en établir le lien important avec l'intervention auprès de personnes vivant avec le VIH ou PVVIH ${ }^{2}$. 


\section{Tout a commencé...}

En intervention sociale, il est inévitable qu'une problématique nous touche plus qu'une autre et pique davantage notre curiosité. C'est en septembre 2008, alors que je débutais mon deuxième stage de Baccalauréat en service social au Bureau régional d'action sida (BRAS) à Hull que j'ai pour la première fois été confrontée à la problématique du VIH/sida. J'en étais à ma dernière session en tant qu'étudiante au premier cycle et ne me doutais pas que cette expérience allait marquer le début d'un long parcours dans cette réalité qui m'était auparavant inconnue. Lors de ce stage, j'ai rapidement pris conscience de ma méconnaissance de cette maladie et du vécu des personnes qui en sont atteintes. Mon mandat comme stagiaire au BRAS était d'organiser la semaine de sensibilisation auVIH/sida et de développer des activités visant à sensibiliser et à éduquer les gens sur cette maladie. Pour remplir ce mandat, il était donc essentiel pour moi de suivre la formation de base et de lire divers articles sur le sujet.

Le virus de l'immunodéficience humaine (VIH) s'attaque aux cellules du système immunitaire, plus précisément aux CD4. Lorsque le VIH n'est pas traité, il continue de se multiplier et plus il se multiplie, plus le système immunitaire s'affaiblit. La plupart des personnes infectées par leVIH vivent en santé pendant plusieurs années avant que les premiers symptômes apparaissent. C'est pourquoi plusieurs personnes infectées n'arrivent jamais à déterminer le moment précis où elles l'ont été. La phase sida (syndrome d'immunodéficience acquise) a lieu lorsque le système immunitaire de la personne atteinte est presque complètement épuisé. À cause de la fragilité de leur système immunitaire lors de la phase sida, les personnes infectées sont plus à risque de contracter des maladies opportunistes graves et il leur sera plus difficile de lutter contre elles. De nos jours, grâce à l'efficacité des médicaments anti-VIH (antirétroviraux), de moins en moins de personnes infectées passent au stade sida et pour celles qui s'y retrouvent, les médicaments anti-VIH permettent de rétablir le système immunitaire, et donc, de s'en sortir. Les antirétroviraux 
utilisés pour traiter le VIH le sont sous forme de multithérapies afin de s'attaquer au virus sous différents angles ${ }^{3}$. De cette manière, ils sont beaucoup plus efficaces.

\section{Les représentations de la maladie : morales, biologiques et politiques}

Encore en 2012,leVIH/sida reste une maladie méconnue autour de laquelle se sont développés divers préjugés et stigmatisations. Le VIH/sida est souvent associé à des comportements dits « de déviants " - homosexualité et utilisation de drogues injectables - et beaucoup de gens croient encore qu'il s'agit d'une maladie mortelle. Mais d'où viennent cette méconnaissance et ces préjugés? En $1981^{4}$, alors que le VIH/sida faisait ses premières victimes en Amérique du Nord, la communauté médicale a été mise au défi par cette maladie que personne ne semblait comprendre et encore moins en mesure d'expliquer. La première réponse médiatique est à la base de diverses représentations sociales du VIH/sida encore en vogue aujourd'hui. Du fait que les premières victimes avaient toutes été des homosexuels ${ }^{5}$, les premiers discours portant sur le VIH ancraient cette maladie dans la communauté gaie.

Les premiers articles scientifiques publiés se référaient au Gay Related Immunodeficiency Disease ou à « la peste gaie ». Ces articles n'ont fait qu'encourager les médias populaires à utiliser ce même langage et la communauté gaie a été identifiée comme étant le seul groupe à risque. Quelques mois après que les premiers cas de sida furent diagnostiqués aux États-Unis, une deuxième vague de victimes a été identifiée. Mais ces dernières appartenaient cette fois à un groupe différent, les utilisateurs de drogues injectables (UDI), la cause principale de la forte incidence de VIH/sida chez eux étant le partage de matériel d'injection. Pour ces deux groupes de personnes infectées, la maladie était donc un signe de marginalité, voire d'exclusion. Ce sont ces personnes qui, les premières, ont donné visage à la maladie. 
En transmettant cette information, les médias ont fait en sorte que la population se créé de la maladie une représentation ne relevant que de ces deux groupes. Conséquemment, les hétérosexuels ne se sentaient pas concernés par la maladie, les homosexuels comme les UDI étaient rejetés encore plus par la société et le VIH/sida a été perçu comme punition de comportements « de déviants».

Les représentations du VIH/sida ont donc été grandement influencées par les discours tant scientifiques que médiatiques. S'ajoutant aux représentations d'ordre moral, les discours du monde médical n'ont fait que semer de la panique dans l'espace public, car aucune explication sur les origines du VIH/sida ou sur ses modes de transmission n'avait été avancée. Ce manque de connaissances a aussi eu comme effet de semer la méfiance et la peur dans la société, car en ne connaissant pas les modes de transmission, on identifiait les personnes infectées comme étant un danger pour les autres. On les considérait comme des facteurs de propagation de la maladie.

Depuis 1981, les connaissances sur le VIH/sida ont beaucoup évolué. La cause du sida - leVIH — et ses modes de transmission sont aujourd'hui connus et divers médicaments sont disponibles. L'avancement des connaissances a fait en sorte qu'aujourd'hui, les PVVIH connaissent une meilleure qualité de vie et une plus grande espérance de vie. Mais il reste que vivre avec leVIH signifie avoir à affronter les divers préjugés et stigmatisations entourant encore la maladie. Qu'une personne séropositive puisse mettre au monde un enfant reste impensable pour la majorité des gens, peu ou mal informés des nombreux visages du VIH/sida.

\section{Une expérience de la maladie : donner la vie, le parcours de mères séropositives}

Mon mémoire de maitrise porte sur une recherche menée auprès de femmes séropositives d'origines africaines qui ont choisi de vivre une grossesse après avoir découvert leur séropositivité. Cette 
étude a cherché à comprendre dans quelle mesure le choix de faire un enfant représentait pour elles une libération, un processus de normalisation et de protection. En ancrant ma recherche dans l'approche phénoménologique, il m'a été possible de m'infiltrer dans le vécu de ces femmes afin de comprendre leur expérience de grossesse. L'approche phénoménologique permet de comprendre ce que l'autre vit en lui donnant une voix et en interprétant son témoignage. Dans le contexte du VIH/sida, un bon nombre de PVVIH choisissent de taire leur infection; en conséquence, très peu témoignent de leur expérience. Cette approche m'a permis de recueillir auprès de trois femmes les données nécessaires à analyser leur expérience de grossesse; elle m'a aussi permis de découvrir un visage du VIH/sida qui m'était jusque-là méconnu, à savoir, la réalité du VIH/sida en Afrique subsaharienne ${ }^{6}$.

L'Afrique subsaharienne est la région la plus touchée par le $\mathrm{VIH} / \mathrm{sida}^{7}$.Et dès les débuts de l'épidémie en Amérique du Nord, l'Afrique subsaharienne en a été identifiée comme responsable. Les pays industrialisés touchés par cette épidémie condamnaient les pratiques ainsi que les croyances des Africains et stipulaient que leur promiscuité et leurs pratiques sexuelles immorales étaient à la source du sida. Ce regard que les pays industrialisés posaient sur l'Afrique a eu comme effet de développer divers préjugés, autant à l'intérieur qu'à l'extérieur de ce continent ${ }^{8}$.

Tandis qu'en Amérique du Nord, les représentations duVIH/ sida associent cette maladie à des comportements de déviants, en Afrique subsaharienne cette maladie est associée à la sorcellerie et au châtiment divin. Cela fait en sorte que les personnes infectées faisant partie au Canada d'une communauté d'origine subsaharienne sont confrontées à diverses représentations de la maladie, ce qui rend encore plus difficile l'annonce de leur diagnostic à leur entourage.

Quatre grands thèmes se dégagent des données recueillies lors des entrevues individuelles avec les participantes : l'infection; le secret; le désir d'enfants; le monde médical. Leurs récits sont empreints de douleur, de courage et d'espérance. 
Ces femmes racontent le viol qui les a contaminées et les émotions intenses que leur fait revivre le souvenir de cet événement tragique, entre autres, l'abandon de la part de leurs proches, la peur d'être rejetée, la honte, la culpabilité et la peur de mourir. Elles nous apprennent que les préjugés et stigmatisations entourant le VIH/sida font que le secret du diagnostic est souvent plus facile à supporter que le rejet et l'ostracisme provenant de leur entourage. Le secret — leur lieu de refuge — les protège et protège également leur mari et leurs enfants. Même si elles sont protégées de la stigmatisation de "mauvaises femmes " dont elles seraient victimes dans leur pays en tant que séropositives, ici, ces femmes restent marquées par la honte; alors, elles se taisent, car parler de leur séropositivité signifie également devoir parler de la façon dont elles ont été infectées. Les seules personnes de leur entourage qui sont informées de leur séropositivité sont les membres de leur famille immédiate (parents, frères ou sœurs, mari et enfants).

Par ailleurs, ces femmes résilientes, fortes et déterminées racontent qu'avec le poids du secret et du rejet qui les guette si leur condition se sait, elles ont vu grandir en elles le désir d'avoir des enfants. Se croyant condamnées à mourir en apprenant leur séropositivité, elles ont par la suite décidé de devenir mères. Pour elles, avoir un enfant signifiait mettre fin à l'isolement et à la solitude dans laquelle la maladie les avait plongées. Pour elles, leur grossesse les protégeait également des stigmatisations associées au VIH/sida, car très peu d'individus savent qu'il est possible pour une femme séropositive de vivre une grossesse sans transmettre le VIH à son partenaire ou au foetus. Pour Ava, Marie et Celeste, ce désir d'enfant a été vécu en couple et elles savent que leur mari et leurs enfants ne les abandonneront jamais. Ces enfants représentent l'espoir de ces femmes; l'espoir de vivre une vie normale et de voir leurs enfants grandir.

Alors que ces femmes n'ont pas annoncé leur séropositivité à leur réseau, c'est le monde médical dans lequel elles sont plongées qui les a accompagnées dans cette étape importante de leur vie. En acceptant le recours à la biotechnologie, elles ont été suivies durant les neuf mois de leur grossesse par une 
équipe de soins qui a réussi, à de multiples reprises, à apaiser leurs inquiétudes. Masquant à leur réseau un pan de leur vie de peur des conséquences qu'engendrerait le dévoilement de leur condition, elles se sont senties acceptées dans leur intégrité par les professionnels de la santé.

Pour clore cette section sur l'expérience de maternité de femmes séropositives, rappelons que la grossesse s'est avérée un processus de normalisation, un moyen de libération et une forme de protection pour Ava, Marie et Celeste.

Malgré leur séropositivité, ces femmes ont pu devenir mères et avoir des enfants en santé. Le monde médical qui les a accompagnées dans cette étape de leur vie les a traitées comme des femmes «normales» et leur équipe de soins a fait tout ce qu'elle pouvait afin de diminuer les risques de transmission verticale et horizontale ${ }^{9}$. La naissance de leurs enfants constitue pour elles une forme de normalisation, car la plupart des gens de leur entourage ignorent qu'il est possible pour une femme séropositive de vivre une grossesse et d'avoir des enfants en santé.

Cette expérience de maternité a aussi été un moyen de libération : elle a libéré ces femmes du diagnostic de «mort » qu'elles croyaient avoir reçu. Leur grossesse les a amenées à penser un peu moins aux dommages que le VIH/sida leur causerait à l'avenir; elles ont appris à vivre un peu plus le moment présent. Le choix d'avoir des enfants leur a permis de reprendre un contrôle sur leur vie. Tandis que leur infection au $\mathrm{VIH} /$ sida a été le résultat d'un évènement sur lequel elles n'avaient aucun contrôle, leur désir d'enfants et leur expérience de grossesse en sont tout le contraire.

Pour ces femmes, leur grossesse a constitué enfin une forme de protection. Les diverses possibilités qui leur ont été offertes par le monde médical leur ont permis de minimiser les risques de transmission. Ces femmes, qui ne pensaient jamais pouvoir porter en elles un enfant, ont vécu une grossesse qui a été synonyme de "pouvoir " sur leur vie. Cette expérience de maternité leur a démontré qu'il leur est quand même possible de vivre la vie comme elles l'ont toujours souhaité. 


\section{Pour l'intervention : l'accompagnement de personnes vivant avec le VIH}

Considéré au départ comme une maladie infectieuse mortelle, le VIH/sida est aujourd'hui une maladie chronique. Cela fait en sorte que les personnes séropositives sont suivies par leur équipe de soins pendant plusieurs années. En tant que travailleur social, une grande écoute est nécessaire, car l'accompagnement à long terme nécessite la création de liens de confiance. Pour plusieurs PVVIH, une grande partie de leur entourage ne sera pas mise au courant de leur séropositivité; donc, les rencontres avec le travailleur social sont souvent le seul endroit où verbaliser leurs inquiétudes.

Lors de mes interventions auprès de personnes nouvellement diagnostiquées avec le VIH/sida, j'ai constaté qu'il leur était difficile d'accepter ce diagnostic alors qu'elles n'en ressentaient aucun des symptômes. Il est encore plus difficile pour elles de retracer la personne ou les événements responsables de leur état. Pour plusieurs PVVIH, ce questionnement dure plusieurs années et reste même sans réponse. Les représentations du VIH/ sida qui ont eu comme effet de lier cette maladie à des pratiques de déviants ou bien au châtiment divin ou au péché rendent encore plus difficile l'acceptation de cette maladie. Plusieurs des personnes nouvellement diagnostiquées éprouvent de la honte ou de la culpabilité et mettent parfois des années avant d'en arriver à l'accepter; pour d'autres, il sera difficile de commencer la prise de médicaments alors qu'elles se sentent tout à fait normales et en santé.

Afin d'aider les PVVIH à accepter leur diagnostic, mais surtout à mieux comprendre le VIH/sida, il est primordial pour les travailleurs sociaux œuvrant dans le domaine de maitriser les connaissances de base sur la maladie et de les tenir à jour. Au moment du diagnostic, le médecin spécialiste transmet diverses informations à son patient; mais vu l'état de choc dans lequel il se trouve, ce dernier ne peut pas se rappeler toute cette 
information. Le travailleur social a souvent à démystifier les savoirs de la personne sur le VIH/sida. Au fil des rencontres, beaucoup d'information est transmise, mais à petites doses, afin de donner à la personne le temps nécessaire à l'intégrer.

Avec toute la méconnaissance et les préjugés entourant leVIH/ sida, il se peut qu'une PVVIH demande à son travailleur social de l'accompagner lors de l'annonce de sa séropositivité à son entourage. Dans cette situation, le travailleur social a pour tâche principale de renseigner les membres de l'entourage sur les réalités du VIH/sida et de diminuer leurs inquiétudes en répondant à leurs questions. Il se peut également que le travailleur social ait à réconforter la personne atteinte si l'annonce du diagnostic provoque des réactions négatives de la part de membres de son entourage.

Lors de mon stage au BRAS et de mon stage de maîtrise à la clinique d'immunodéficience (Module G) de l'Hôpital d'Ottawa, j'ai eu l'occasion de rencontrer de très nombreuses PVVIH, entre autres, des femmes infectées par un mari infidèle, des UDI infectés en partageant de matériel d'injection, un homme trompé par son partenaire infecté à la suite d'une relation non protégée, une prostituée ayant fait un peu plus d'argent avec un client qui ne voulait pas porter le condom. Alors que les premières victimes du $\mathrm{VIH} /$ sida étaient principalement des homosexuels et des UDI, le nombre de personnes infectées à la suite d'une relation hétérosexuelle a beaucoup augmenté depuis les débuts de l'épidémie. Et l'âge des personnes nouvellement infectées varie de plus en plus ${ }^{10}$. Malgré ces variantes, les émotions vécues par les personnes infectées demeurent les mêmes. Plusieurs vivent un repli sur elles-mêmes de peur d'être rejetées et confrontées aux préjugés et discriminations de leur entourage.

Lors de mon stage au module G, ma superviseure de stage m'encourageait à utiliser le toucher lors de mes interventions. C'est que beaucoup de ses patients avaient été victimes de rejet de la part de leur entourage à l'annonce de leur séropositivité. De là, toute l'importance du toucher dans les interventions. Ce geste indique qu'il n'y a aucun risque de transmission par une simple caresse ou un baiser sur la joue, et que le réconfort que cela procure ne leur est pas retiré avec le diagnostic. 
L'histoire du VIH/sida est balisée de progrès sur le plan médical et depuis 1981, le portrait des personnes infectées a beaucoup changé. Parce qu'il s'agit d'une maladie relativement récente, de nouvelles problématiques touchant les PVVIH font surface d'année en année. Par exemple, les personnes qui ont été diagnostiquées au tout début de l'épidémie, et qui ont été médicamentées, sont aujourd'hui âgées d'une cinquantaine ou soixantaine d'années. Or, plusieurs d'entre elles vivent des changements dans leur état de santé et leur équipe de soins ignore si ceux-ci sont attribuables au VIH ou bien à la ménopause ou à l'andropause. De nouvelles recherches sont en cours afin d'étudier les effets des changements hormonaux sur la santé des PVVIH et de voir comment ces changements affectent la médication ainsi que la progression du virus.

Il y a également la première vague d'adultes infectés durant la grossesse de leur mère, soit avant la mise en place de lignes directrices visant à prévenir l'infection verticale. La séropositivité de ces jeunes adultes affectera leurs relations sexuelles; il est de plus possible que l'observance d'un traitement ait été négligée durant leur adolescence. Ces jeunes adultes bénéficieront de divers moyens médicaux leur permettant un jour d'avoir des enfants séronégatifs. Même si l'épidémie du VIH/sida peut être perçue comme étant jeune, il n'en reste pas moins qu'en trente ans, la communauté mondiale en a dévoilé de multiples visages et qu'encore aujourd'hui, des enjeux surgissent à son sujet. Il est nécessaire de donner à ces jeunes adultes nés avec leVIH/sida un espace pour s'exprimer. Il se peut que leur crise d'adolescence les amène à se replier sur eux-mêmes plutôt que de parler de leurs émotions. Dans un tel cas, le travailleur social peut les encourager à utiliser un autre moyen pour s'exprimer, par exemple, le dessin, l'écriture ou la photographie.

Leur étant difficile de parler de leur séropositivité à leur entourage, ces jeunes adultes pourraient rencontrer, dans le cadre de groupes de soutien, d'autres individus vivant avec leVIH/sida. Un groupe de soutien ouvert à tous les groupes d'âge pourrait les aider à accepter leur séropositivité et leur donner de l'espoir face à l'avenir. Un groupe de soutien basé sur la notion du «par 
et pour " leur permettrait de réaliser que leur diagnostic ne les empêche nullement de prendre le contrôle sur leur vie.

Un groupe de soutien leur permettrait enfin de discuter de sujets qui les concernent tous et de créer des liens avec des gens qui vivent la même réalité qu'eux. Au Comité sida d'Ottawa (CSO), un groupe de soutien rassemble des femmes venant d'Afrique et des Caraibes. Rencontrer d'autres femmes vivant les mêmes difficultés leur a permis de briser leur isolement. Une solidarité s'est rapidement bâtie entre ces femmes séropositives et de nouvelles amitiés se sont développées.

Pour mettre fin à la méconnaissance populaire face au VIH/ sida ainsi qu'aux préjugés et stigmatisations qui en découlent, il est important d'éduquer, d'informer et de sensibiliser les gens. Divers organismes œuvrant dans la problématique du VIH/ sida offrent non seulement des services aux PVVIH et à leur entourage, mais organisent également des activités d'éducation et de sensibilisation. C'est en éduquant les gens sur la réalité duVIH/ sida et en les sensibilisant que la société en viendra à accepter les PVVIH. Cette acceptation permettra à ces derniers, entre autres, d'annoncer leur séropositivité à leur entourage sans en craindre les répercussions.

\section{En guise de conclusion...}

Mon engagement dans la problématique duVIH/sida m'a permis non seulement d'en apprendre sur la réalité sociale et médicale de cette maladie, mais également de m'entretenir avec plusieurs PVVIH et de découvrir comment se vit cette réalité. J'ai autant appris des témoignages des répondantes à ma recherche et des usagers des services que des conférences et formations auxquelles j'ai assisté et des divers rapports de recherches et articles que j'ai consultés. Malgré un parcours long de trente ans, il reste beaucoup à accomplir, tant sur le plan médical que social, pour mettre fin à l'épidémie du VIH/sida. Si la découverte d'un vaccin demeure imminente, les préjugés et stigmatisations, eux, sont encore bien réels. À nous tous d'y voir! 


\section{Notes}

1 Une attention particulière doit être portée à l'accompagnement offert par Marguerite Soulière, ma directrice de mémoire. Par ses patients commentaires, elle m'a guidée tout au long de la rédaction de mon mémoire ainsi que du présent article.

2 Le terme maintenant utilisé pour parler des personnes atteintes duVIH/sida est "personne vivant avec leVIH/sida » (PVVIH), car avec la chronicité de la maladie, les personnes qui reçoivent un diagnostic de séropositivité vivent maintenant avec la maladie sans risque de mourir du sida.

3 Les différents médicaments utilisés lors de multithérapies permettent non seulement de restaurer le système immunitaire, mais également de ralentir la multiplication duVIH dans le corps et de maitriser le virus.

4 À la fin 2010, on estimait à 34 millions [31,6-35,2 millions] le nombre de PVVIH dans le monde (ONUSIDA, 2011, p. 6). Selon l'Agence de santé publique du Canada (2010), on estimait que 65000 Canadiens vivaient avec le VIH/sida en 2008.

5 C'est à la suite de la découverte de cinq cas de pneumocystose pulmonaire, une forme de pneumonie qui apparait lorsque le système immunitaire est complètement épuisé, que le sida s'est premièrement manifesté en Amérique du Nord. Suivront des cas de sarcome de Kaposi, une forme de cancer qui se manifeste essentiellement chez les personnes dont le système immunitaire est affaibli. Malgré le fait que les cas de pneumocystose et de sarcome de Kaposi étaient diagnostiqués chez des hommes homosexuels, aucun lien de causalité ne pouvait être détecté chez ces individus.

6 Avant même que les premiers cas de sida se soient manifestés en Amérique du Nord, des rapports signalaient depuis plusieurs années la présence en Afrique d'une maladie, le slim disease — ainsi nommée parce que les personnes qui en étaient atteintes perdaient beaucoup de poids. «Des milliers d'Africains sont déjà morts de ce qu'on appellera plus tard le sida, alors que la maladie débute officiellement par l'identification des premiers cas caractéristiques chez les Blancs " (Bourdillon et Sobel, 2006, p. 54).

7 Selon ONUSIDA (2011, p. 6), des 34 millions d'individus vivant avec leVIH dans le monde en 2010, $68 \%$ résident en Afrique subsaharienne.

8 Afin de détourner l'attention de l'Afrique subsaharienne, les communautés africaines ont essayé de trouver les vrais coupables du VIH/sida. En Afrique subsaharienne, ce sont les prostituées qui ont été identifiées comme étant les "vraies responsables " de cette maladie. Tout comme dans les pays industrialisés, les pays d'Afrique subsaharienne touchés par leVIH/sida n'arrivaient pas à trouver les origines de cette maladie et possédaient peu d'information sur ses modes de transmission. «Plus l'épidémie du sida s'étend en Afrique du Sud, plus les soupçons de sorcellerie éclosent sur son passage, de sorte qu'elle devient ainsi une épidémie de sorcellerie " (Ashfort, 2002, p. 119). La maladie est également qualifiée de punition divine, infligée à un individu ayant péché : "Une personne est malade du sida parce qu'elle a fait "quelque chose de mal”, comme de ne pas "se respecter soi-même" (vie "immorale", souvent associée aux influences occidentales), ou "qu'elle s'est mal comportée" dans ses affaires; ou alors elle a offensé les ancêtres, ou a été 
ensorcelée, à la suite de jalousie. Il y a des témoignages, des "explications" de ce genre venant de tout le continent" (Seidel et Capitan, 1996, p. 53).

9 Le terme "transmission verticale " est utilisé pour la transmission de la mère séropositive à l'enfant. Le terme "transmission horizontale » est utilisé pour la transmission du VIH d'une personne séropositive à sa partenaire ou à son partenaire.

10 Selon l'Agence de la santé publique du Canada (2010), « Depuis les dix dernières années, 39,9\% des cas de sida diagnostiqués chez les femmes étaient associés au groupe d'âge des 30 à 39 ans, ce qui est comparable à la moyenne de ce groupe de 1985 à 1998. Depuis les dix dernières années, on a toutefois constaté un changement important en ce qui concerne la proportion de cas de sida diagnostiqués dans le groupe d'âge des 20 à 29 ans, la moyenne ayant chuté à 17,9\%, soit $6,5 \%$ de moins que la moyenne historique de ce groupe pendant la durée de l'épidémie. En revanche, la proportion de cas de sida diagnostiqués dans le groupe d'âge des 40 à 49 ans a augmenté à $28,3 \%$ chez les femmes, ce qui correspond à une augmentation de 7,1\% par rapport à la moyenne historique ".

\section{Bibliographie}

AGENCE DE LA SANTÉ PUBLIQUE DU CANADA (2009). «Le VIH et le sida au Canada : rapport de surveillance en date du 31 décembre 2009 ", réf. du 12 avril 2012, http://www. phac-aspc.gc.ca/aids-sida/publication/survreport/2009/dec/pdf/2009-Report-Rapport.pdf

ASHFORT,Adam (2002). "Quand le sida est sorcellerie - Un défi pour la démocratie sur-africaine », Critique internationale, Vol. 1, No 14, p. 119-141.

BOURDILlON, François, et Alain SOBEL (2006). "L'épidémie de sida : le temps des transformations ", Les Tribunes de la santé,Vol. 4, No 13, p. 53-67.

DESCLAUX, Alice (2002). "Stigmatisation, discrimination : que peut-on attendre d'une approche culturelle? ", UNESCO, L'approche culturelle de la prévention et du traitement du VIH/sida - Projet de recherche UNESCO/ONUSIDA VIH/sida, Paris, 2003, p. 1-9.

FORTIN, Marie-Céline (2001). "LeVIH-sida au féminin : Impact du diagnostic de séropositivité sur les conditions de vie des femmes hétérosexuelles de Québec ", Les cahiers de la femme, Vol.21, $\mathrm{N}^{\circ} 12$, p. $20-24$.

JODELET, Denise (1997). Les représentations sociales, Paris, Presses universitaires de France, 447 p.

MASSÉ, Raymond (1995). «Les interprétations et les explications de la maladie : le savoir populaire en tant que système culturel ", Culture et santé publique, Boucherville, Les Éditions Gaëtan Morin, p. 275-312.

MENSAH, Maria Nengeh (2003). Ni vues ni connues? Femmes, VIH, Médias, Montréal, Les éditions du remue-ménage, $221 \mathrm{p}$.

ONUSIDA (2011). "Journée mondiale sida 2011 : Rapport ONUSIDA ", ONUSIDA, réf. du 12 avril 2012, http://www.unaids.org/en/media/unaids/contentassets/documents/ unaidspublication/2011/JC2216_WorldAIDSday_report_2011_fr.pdf

ROUTY, Jean-Pierre (2011). Ce que le sida a changé, Montréal, Héliotrape, 154 p.

SEIDEL, Gill, et Colette CAPITAN (1996). "La représentation des femmes dans le discours sur le sida en Afrique subsaharienne ", Mots, Vol. 49, No 1, p. 48-70. 